\title{
Correction of Transducers Mass Effects from the Measured FRFs in Hammer Impact Testing
}

\author{
Jun Ren, ${ }^{1}$ Jun Wang, ${ }^{1}$ and Shusheng $\mathrm{Bi}^{2}$ \\ ${ }^{1}$ School of Mechanical Engineering, Hubei University of Technology, Wuhan, Hubei 430068, China \\ ${ }^{2}$ Robotics Institute, Beihang University, Beijing 100091, China \\ Correspondence should be addressed to Jun Ren; renjun@mail.hbut.edu.cn
}

Received 23 July 2017; Revised 28 September 2017; Accepted 16 October 2017; Published 13 November 2017

Academic Editor: Matteo Filippi

Copyright (c) 2017 Jun Ren et al. This is an open access article distributed under the Creative Commons Attribution License, which permits unrestricted use, distribution, and reproduction in any medium, provided the original work is properly cited.

\begin{abstract}
In modal testing, the measured frequency response functions (FRFs) are often affected by transducer mass effects. Especially when multiple sensors are employed, transducers mass effects are more significant. This paper deals with removing sensors mass effects from the measured FRFs in hammer impact testing. The proposed method offers some advantages over the available techniques in that extra FRFs measurements with different configurations are not required during the correction process. Firstly, correction method for single accelerometer case is discussed. Further, general correction formulation for multiple accelerometers case is derived. Then, validations of the proposed method are demonstrated using simulated and experimental data. It shows good effectiveness in experimental validation for single accelerometer case. Numerical simulation also demonstrates a good performance for multiple accelerometers case. However, further simulations with noisy-polluted data show that the accuracy of correction results will be affected to some extent by the noise. It is suggested that the measured FRFs be preprocessed using the curve-fitting procedure or noise reduction processing before applying the proposed method.
\end{abstract}

\section{Introduction}

Frequency response functions (FRFs) are widely utilized in structural dynamics analysis and modification. However, accuracies of the measured FRFs are usually affected due to a variety of factors, one of which is transducer mass effect $[1,2]$. For large-scale test structures, mass effect of the sensor is relatively small and it is usually artificially ignored in the test. But for lightweight or small-scale structure, mass effect introduced by the sensor is notable [3, 4]. Especially when multiple sensors are employed, transducers masses loading effects are significant. The measured FRFs may contain larger errors. It is thus necessary to correct the measured FRFs before they are utilized for further analysis.

In early, a mass effects correction method for driving point FRF was introduced by Ewins [1]. Afterwards, some publications focused on removing such effects from transfer FRFs. Decker and Witfeid [5] presented a method to correct the transducer mass effects from transfer FRFs based on substructuring techniques. Ashory [6] proposed a method in which transfer FRFs can be corrected if the measurement is repeated with an accelerometer of different mass. Cakar and Sanliturk [7] presented a correction method by employing dummy mass. Ren et al. $[8,9]$ proposed a correction method for eliminating accelerometer and force transducer mass loading effects from measured FRFs in shaker modal testing. These researches mainly focus on removing mass effects of individual sensor. And extra FRFs measurements with different configurations are needed in the process of correcting the measured transfer FRFs, such as moving the sensor to different response locations, employing accelerometers of different masses or adding extra accelerometer (or dummy mass) on the structure. Zamani et al. [10] presented a strategy for correcting accelerometer mass effect using sensitivity analysis and the effect of accelerometer's mass can be neglected using this strategy. Yin [11] presented a method for eliminating transducer additional mass effects in timedomain responses.

In practical modal test, it usually required to install multiple sensors for measuring some certain FRFs. The current authors presented a method for correcting multiple transducers mass effects from the measured FRFs in 


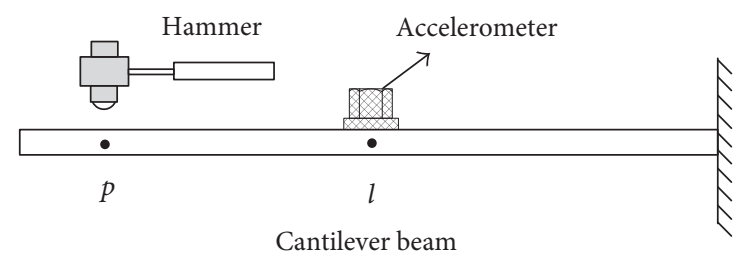

FIGURE 1: Hammer impact testing of cantilever beam.

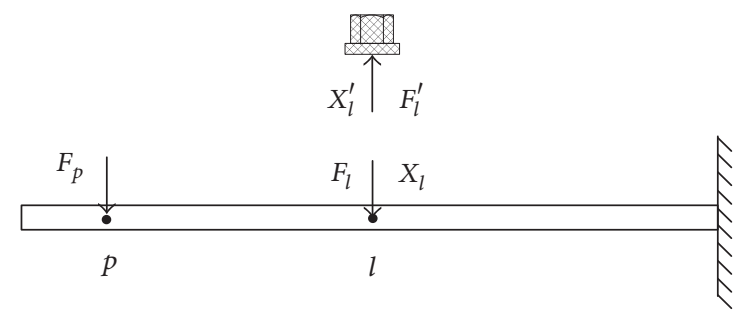

Figure 2: Free body diagram of the system.

an earlier work [12]. This article expands on that work with differences on the following: (1) correction method for both single accelerometer and multiple accelerometers cases are discussed; (2) experiments validation, analysis, and discussions are supplemented except for numerical case study. Firstly, correction method for single accelerometer case is discussed. Further, general correction formulation for multiple accelerometers case is derived. Then, validations of the proposed method are demonstrated using simulated as well as experimental data. The proposed method offers some advantages over the available techniques in that extra FRFs measurements with different configurations are not required in the process of correcting the measured FRFs. "Different configurations" here means that test structure is changed due to movement of sensors to different response locations or addition of extra sensors (or dummy masses) on the structure.

\section{Correction of Mass Effects of Single Accelerometer from the Measured FRFs}

Hammer impact test of a cantilever beam is shown in Figure 1. The beam is excited at point $p$ by a hammer. And an accelerometer with a mass of $m_{a}$ is mounted at point $l$ to measure the response. Then dynamics of the beam are changed due to accelerometer mass effects.

To facilitate the analysis, accelerometer is separated from the original system, as shown in Figure 2. If excitation force and reaction force of the added masses at point $j$ are $F_{p}$ and $F_{l}$, displacement of point $l$ can be expressed as

$$
X_{l}=H_{l p} F_{p}+H_{l l} F_{l} .
$$

Displacement of accelerometer can also be established by using the receptance and reaction force $F^{\prime}$ :

$$
X_{l}^{\prime}=-\frac{1}{m_{a} \omega^{2}} F_{l}^{\prime}
$$

Constraint equations of forces and displacements at $l$ are

$$
\begin{gathered}
F_{l}=-F_{l}^{\prime} \\
X_{l}=-X_{l}^{\prime} .
\end{gathered}
$$

By substituting (2) and (3) into (1), ones yields

$$
H_{l p}^{(l)}=\frac{X_{l}}{F_{p}}=\frac{H_{l p}}{1+m_{a} \omega^{2} H_{l l}},
$$

where $H_{l p}^{(l)}$ is measured FRF (with mass effects). And $H_{l p}$ and $H_{l l}$ are exact FRFs (without mass effects). FRFs in (4) are receptances. In practical test, however, accelerances are utilized more frequently. This poses no difficulty since receptances and accelerances are just two different forms of presenting the same FRF. Multiplying both sides by $-\omega^{2}$ of (4), one yields

$$
A_{l p}^{(l)}=\frac{A_{l p}}{1+m_{a} A_{l l}},
$$

where $A$ denotes accelerance. If excitation point is moved from $p$ to $l$, accelerance of point $l$ can be easily obtained as

$$
A_{l l}^{(l)}=\frac{A_{l l}}{1+m_{a} A_{l l}} .
$$

By rearranging (5) and (6), correction formulas of measured accelerances can be obtained as follows:

$$
\begin{aligned}
A_{l l} & =\frac{A_{l l}^{(l)}}{1-m_{a} A_{l l}^{(l)}} \\
A_{l p} & =\frac{A_{l p}^{(l)}}{1-m_{a} A_{l l}^{(l)}} .
\end{aligned}
$$

It can be seen from (7) and (8) that the measured accelerances can be corrected if both point and transfer accelerances are measured.

\section{Theoretical Development of the Method for Multiple Accelerometers Case}

An $N$ dof mass-spring system is presented in Figure 3(a). To measure the responses, $N$ accelerometers with masses $\Delta m_{1}, \Delta m_{2}, \ldots, \Delta m_{n}$ are installed on point $1,2, \ldots, N$, respectively. Then the system is changed due to additional masses, as it can be seen in Figure 3(b).

To facilitate the analysis, accelerometers are separated from the original system, as shown in Figure 4. The system 


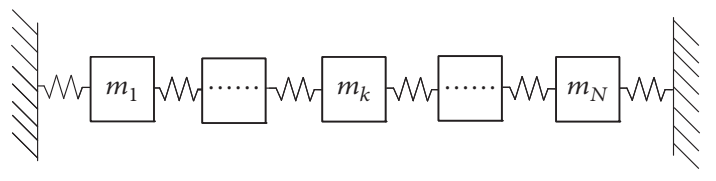

(a) Original system (corresponding to $\operatorname{FRF} \alpha$ )

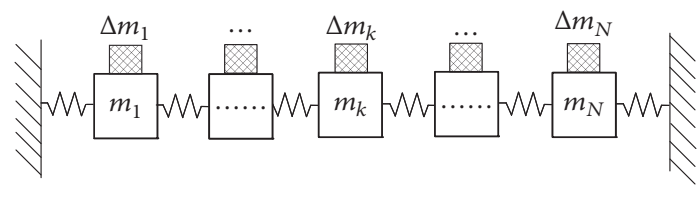

(b) Modified system by adding the extra masses (corresponding to FRF $\alpha^{\prime}$ ).

FIGURE 3: An $n$ dof mass-spring system.

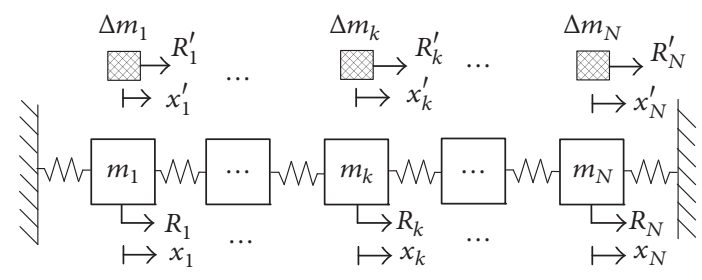

FIGURE 4: Free body diagram of the $n$ dof system.

is excited at point $k$ and the excitation force is $F_{k}$. Then displacements of point $1,2, \ldots, N$ can be expressed as

$$
\begin{gathered}
x_{1}=\alpha_{1 k} F_{k}+\sum_{j=1}^{N} \alpha_{1 j} R_{j} \\
x_{2}=\alpha_{2 k} F_{k}+\sum_{j=1}^{N} \alpha_{2 j} R_{j} \\
\vdots \\
x_{N}=\alpha_{N k} F_{k}+\sum_{j=1}^{N} \alpha_{N j} R_{j},
\end{gathered}
$$

where $x_{1}, x_{2}, \ldots, x_{N}$ are displacements of points 1,2 , and $N$, respectively. $\alpha_{i k}$ is receptance (relating to excitation point $k$ and response point $i$ ) of original system. And $R_{1}, R_{2}$, and $R_{N}$ are reaction forces of the added masses at point $1,2, \ldots, N$, respectively. The constraint equations are

$$
\begin{gathered}
x_{1}=x_{1}^{\prime}, \\
x_{1}=x_{1}^{\prime} \\
\vdots \\
x_{N}=x_{N}^{\prime} \\
R_{1}=-R_{1}^{\prime}, \\
R_{1}=-R_{2}^{\prime} \\
\vdots \\
R_{N}=-R_{N}^{\prime} .
\end{gathered}
$$

The governing equations for the added masses $\Delta m_{1}, \Delta m_{2}$, $\ldots, \Delta m_{n}$ are

$$
\begin{gathered}
H_{11}^{\prime}=\frac{x_{1}^{\prime}}{R_{1}^{\prime}}=-\frac{1}{\Delta m_{1} \omega^{2}} \\
H_{22}^{\prime}=\frac{x_{2}^{\prime}}{R_{2}^{\prime}}=-\frac{1}{\Delta m_{2} \omega^{2}} \\
\vdots \\
H_{N N}^{\prime}=\frac{x_{N}^{\prime}}{R_{N}^{\prime}}=-\frac{1}{\Delta m_{N} \omega^{2}},
\end{gathered}
$$

where $H_{11}^{\prime}, H_{22}^{\prime}$, and $H_{N N}^{\prime}$ are receptances of the added masses. Then the reaction forces of the added masses are

$$
\begin{aligned}
& R_{1}=x_{1} \Delta m_{1} \omega^{2} \\
& R_{2}=x_{2} \Delta m_{2} \omega^{2}
\end{aligned}
$$

$$
R_{N}=x_{N} \Delta m_{N} \omega^{2}
$$

Substituting (12) into (9) yields

$$
\begin{gathered}
x_{1}=\alpha_{1 k} F_{k}+\sum_{j=1}^{N} \alpha_{1 j} x_{j} \Delta m_{j} \omega^{2} \\
x_{2}=\alpha_{2 k} F_{k}+\sum_{j=1}^{N} \alpha_{2 j} x_{j} \Delta m_{j} \omega^{2} \\
\vdots \\
x_{N}=\alpha_{N k} F_{k}+\sum_{j=1}^{N} \alpha_{N j} x_{j} \Delta m_{j} \omega^{2} .
\end{gathered}
$$

Dividing $F_{k}$ by both sides of (13) yields

$$
\begin{gathered}
\alpha_{1 k}^{\prime}=\alpha_{1 k}+\sum_{j=1}^{N} \alpha_{1 j} \alpha_{j k}^{\prime} \Delta m_{j} \omega^{2} \\
\alpha_{2 k}^{\prime}=\alpha_{2 k}+\sum_{j=1}^{N} \alpha_{2 j} \alpha_{j k}^{\prime} \Delta m_{j} \omega^{2} \\
\vdots \\
\alpha_{N k}^{\prime}=\alpha_{N k}+\sum_{j=1}^{N} \alpha_{N j} \alpha_{j k}^{\prime} \Delta m_{j} \omega^{2},
\end{gathered}
$$


where $\alpha$ s are receptances of original system and $\alpha^{\prime}$ s are receptances of modified system (with transducer masses attached). Rearranging (14) by separating $\alpha^{\prime}$ s and $\alpha$ s yields

$$
\begin{aligned}
& {\left[\begin{array}{ccc}
1-\alpha_{11} \Delta m_{1} \omega^{2} & \cdots & -\alpha_{1 N} \Delta m_{N} \omega^{2} \\
\vdots & \ddots & \\
-\alpha_{N 1} \Delta m_{1} \omega^{2} & & 1-\alpha_{N N} \Delta m_{N} \omega^{2}
\end{array}\right]\left[\begin{array}{c}
\alpha_{1 k}^{\prime} \\
\vdots \\
\alpha_{N k}^{\prime}
\end{array}\right]} \\
& =\left[\begin{array}{c}
\alpha_{1 k} \\
\vdots \\
\alpha_{N k}
\end{array}\right] .
\end{aligned}
$$

It shows that the measured FRFs $\alpha_{i k}^{\prime}(i=1 \cdots N)$ can be obtained according to (15) if exact FRFs $\alpha_{i j}(i=$ $1 \cdots N, j=1 \cdots N$ ) are known. However, it is the opposite in the problem of correcting the transducer mass effects that exact FRFs $\alpha_{i j}$ are to be obtained and FRFs $\alpha_{i k}^{\prime}$ are known (by measurement). Obviously, exact FRF $\alpha_{i k}$ cannot be directly obtained according to (15). This paper proposed a method by adding virtual negative masses to solve this problem, as it can be seen in Figure 5. Modification of the structure by adding extra masses is shown in Figure 5(a) which corresponds with (15). In Figure 5(b), however, the modified system (a3) is considered as "original system" (b1). And then the "modified system" can be generated by adding virtual negative masses $-\Delta m_{i}(i=1 \cdots N)$ to point $1 \cdots N$, respectively. The new obtained "modified system" (b3) is equivalent to original system (a1).

The process of modification of the system in Figure 5(a) can be described by employing (15). To describe Figure 5(b), it only requires to replace $\alpha_{i k}^{\prime}(i=1 \cdots N), \alpha_{i j}(i=1 \cdots N, j=$ $1 \cdots N)$, and $\Delta m_{i}(i=1 \cdots N)$ with $\alpha_{i k}(i=1 \cdots N), \alpha_{i j}^{\prime}(i=$ $1 \cdots N, j=1 \cdots N)$, and $-\Delta m_{i}(i=1 \cdots N)$ in (15). The result is shown in the following:

$$
\begin{aligned}
& {\left[\begin{array}{ccc}
1+\alpha_{11}^{\prime} \Delta m_{1} \omega^{2} & \cdots & \alpha_{1 N}^{\prime} \Delta m_{N} \omega^{2} \\
\vdots & \ddots & \\
\alpha_{N 1}^{\prime} \Delta m_{1} \omega^{2} & & 1+\alpha_{N N}^{\prime} \Delta m_{N} \omega^{2}
\end{array}\right]\left[\begin{array}{c}
\alpha_{1 k} \\
\vdots \\
\alpha_{N k}
\end{array}\right]} \\
& =\left[\begin{array}{c}
\alpha_{1 k}^{\prime} \\
\vdots \\
\alpha_{N k}^{\prime}
\end{array}\right] .
\end{aligned}
$$

It can be seen from (16) that exact FRF $\alpha_{i k}(i=1 \cdots N)$ of original system can be obtained given that transducer masses $\Delta m_{i}(i=1 \cdots N)$ and FRFs $\alpha_{i j}^{\prime}$ of modified system are known.
By extending (8), the genera formula for correcting FRFs $\alpha_{i j}^{\prime}$ $(i=1 \cdots N, j=1 \cdots N)$ can be easily deduced in

$$
\begin{aligned}
& {\left[\begin{array}{ccc}
1+\alpha_{11}^{\prime} \Delta m_{1} \omega^{2} & \cdots & \alpha_{1 N}^{\prime} \Delta m_{N} \omega^{2} \\
\vdots & \ddots & \vdots \\
\alpha_{N 1}^{\prime} \Delta m_{1} \omega^{2} & \cdots & 1+\alpha_{N N}^{\prime} \Delta m_{N} \omega^{2}
\end{array}\right]\left[\begin{array}{ccc}
\alpha_{11} & \cdots & \alpha_{1 N} \\
\vdots & \ddots & \vdots \\
\alpha_{N 1} & \cdots & \alpha_{N N}
\end{array}\right]} \\
& =\left[\begin{array}{ccc}
\alpha_{11}^{\prime} & \cdots & \alpha_{1 N}^{\prime} \\
\vdots & \ddots & \vdots \\
\alpha_{N 1}^{\prime} & \cdots & \alpha_{N N}^{\prime}
\end{array}\right] .
\end{aligned}
$$

By rearranging (17), exact FRF $\alpha_{i j}(i=1 \cdots N, j=1 \cdots N)$ can be solved by

$$
\begin{aligned}
& {\left[\begin{array}{ccc}
\alpha_{11} & \cdots & \alpha_{1 N} \\
\vdots & \ddots & \vdots \\
\alpha_{N 1} & \cdots & \alpha_{N N}
\end{array}\right]} \\
& =\left[\begin{array}{ccc}
1+\alpha_{11}^{\prime} \Delta m_{1} \omega^{2} & \cdots & \alpha_{1 N}^{\prime} \Delta m_{N} \omega^{2} \\
\vdots & \ddots & \vdots \\
\alpha_{N 1}^{\prime} \Delta m_{1} \omega^{2} & \cdots & 1+\alpha_{N N}^{\prime} \Delta m_{N} \omega^{2}
\end{array}\right]^{-1} \\
& \cdot\left[\begin{array}{ccc}
\alpha_{11}^{\prime} & \cdots & \alpha_{1 N}^{\prime} \\
\vdots & \ddots & \vdots \\
\alpha_{N 1}^{\prime} & \cdots & \alpha_{N N}^{\prime}
\end{array}\right] .
\end{aligned}
$$

Discussion above refers to receptances $\alpha$. In practical test, accelerances $A$ are utilized more frequently. Multiplying both sides by $-\omega^{2}$ of (18), one yields

$$
\begin{gathered}
{\left[\begin{array}{ccc}
A_{11} & \cdots & A_{1 N} \\
\vdots & \ddots & \vdots \\
A_{N 1} & \cdots & A_{N N}
\end{array}\right]} \\
=\left[\begin{array}{ccc}
1-A_{11}^{\prime} \Delta m_{1} & \cdots & -A_{1 N}^{\prime} \Delta m_{N} \\
\vdots & \ddots & \vdots \\
-A_{N 1}^{\prime} \Delta m_{1} & \cdots & 1-A_{N N}^{\prime} \Delta m_{N}
\end{array}\right] \\
\cdot\left[\begin{array}{ccc}
A_{11}^{\prime} & \cdots & A_{1 N}^{\prime} \\
\vdots & \ddots & \vdots \\
A_{N 1}^{\prime} & \cdots & A_{N N}^{\prime}
\end{array}\right],
\end{gathered}
$$

where $A$ s are accelerances of original system and $A^{\prime}$ s are accelerances of modified system (with mass effects). It is not surprising that (19) will be coincident with (7) and (8) when only one accelerometer is used.

As it can be seen from (16) (19), all the measured FRFs associated with the response points are required for FRFs 


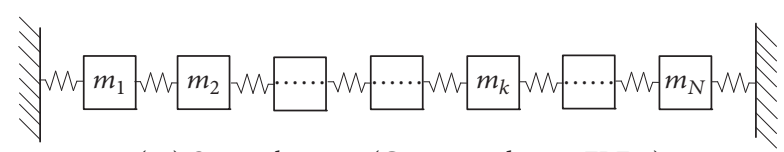

(a1) Original system (Corresponding to FRF $\alpha$ )

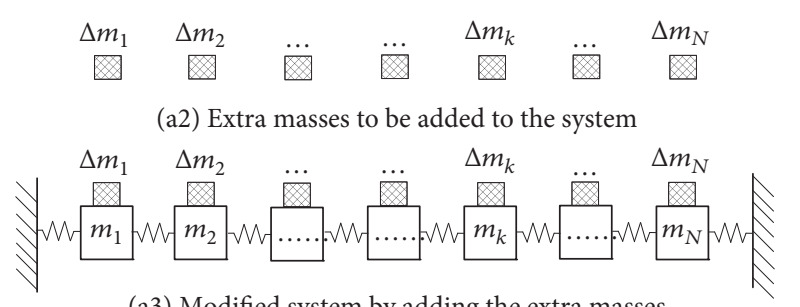

(a3) Modified system by adding the extra masses (Corresponding to FRF $\alpha^{\prime}$ )

(a) Modification of the system by adding extra mass $\Delta m$

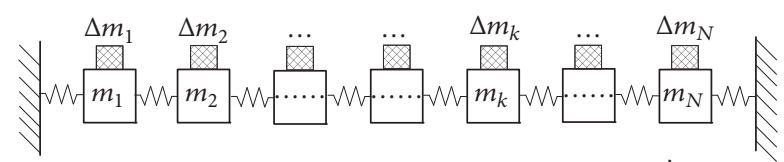

(b1) "Original system" (Corresponding to FRF $\alpha^{\prime}$ )

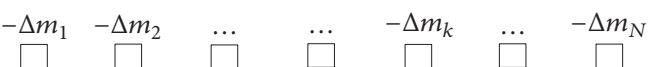

(b2) Adding virtual negative masses at each point

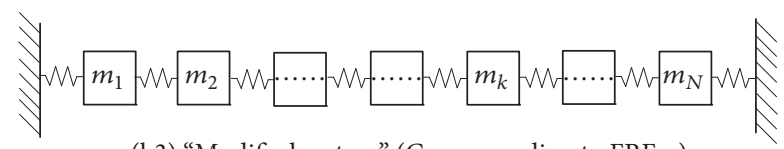

(b3) "Modified system" (Corresponding to FRF $\alpha$ )

(b) Modification of modified system by adding $-\Delta m$

Figure 5: Modification of the system by adding extra masses.

corrections. In the case of $n$ response points, a symmetric matrix consisting of $n^{2}$ FRFs needs to be measured. Since FRF $A_{i j}^{\prime}$ equals $A_{j i}^{\prime}$, only $N(N+1) / 2$ FRFs are required to be measured in fact.

\section{Verification of the Method}

4.1. Experimental Study for Single Accelerometer Case: Impact Testing of a Rectangle Plate. Free-free impact modal testing of a rectangle plate shown in Figure 6 was chosen for the verification of the proposed method. The rectangular plate is suspended by elastic rope, to simulate free-free boundary conditions. The size and weight of the plate are $250 \times 148 \times 6 \mathrm{~mm}^{3}$ and $0.61 \mathrm{Kg}$, respectively. The plate is equipped with 15 measuring points; the acceleration sensor (approximately $8 \mathrm{~g}$ ) is installed at point 2 . Hammer impact is moving sequentially from point 1 to point 15 for measuring accelerances $A_{21}^{(2)}, A_{22}^{(2)}, \ldots, A_{2.15}^{(2)}$. Obviously all the measured FRFs are affected by acceleration sensor mass loading effects. However, only a point FRF $A_{22}^{(2)}$ and a transfer FRF $A_{2.14}^{(2)}$ are chosen to be corrected here for the sake of brevity.

The process of removing accelerometer mass is shown in Figure 7(a). Effectiveness of the proposed method can be assessed by comparing the corrected FRF with the exact

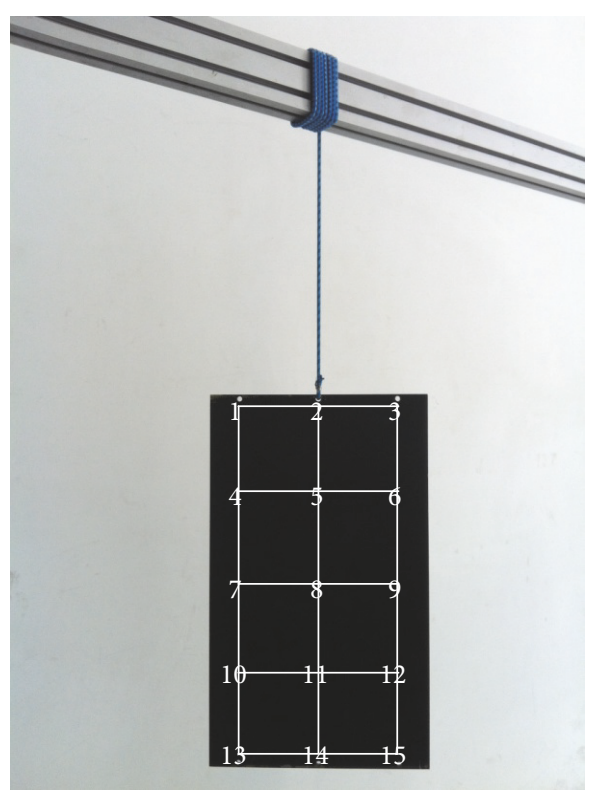

FIGURE 6: Free-free impact modal testing of a rectangle plate.

FRF (Figure 7(a3)). However, exact value is unavailable which makes it difficult to assess. To solve this difficulty, a comparative experiment is designed, as shown in Figure 7(b). An additional mass $m_{\text {ext }}$ is attached point $l$ when accelerance is measured (Figure 7(b1)). And corrected accelerance can be obtained by removing additional mass $m_{\text {ext }}$ from the measured value. Then, the method can be assessed by comparing corrected FRF with target FRF (Figure 7(b3)) which can be measured directly.

According to the idea of comparative experiments, Target FRFs $A_{22}$ and $A_{2-14}$ (without additional mass $m_{\text {ext }}$ ) are firstly measured. Then $A_{22}^{(2)}$ and $A_{2.14}^{(2)}$ (with additional mass $m_{\text {ext }}$ $37 \mathrm{~g}$ ) are measured. Then corrected FRFs $A_{22}^{*}$ and $A_{2-14}^{*}$ can be calculated by employing (7) and (8). Effectiveness of the correction method can thus be assessed by comparing $A_{22}$ and $A_{2-14}$ with $A_{22}^{*}$ and $A_{2.14}^{*}$, respectively.

As shown in Figure 8, resonance frequencies of the measured accelerance $A_{22}^{(2)}$ are lower than those of the target accelerance $A_{22}$ due to extra mass effects. After correction of extra mass $m_{\text {ext }}$, as expected, the frequencies of corrected accelerance $A_{22}^{*}$ as a whole are in quite good agreement with those of the target accelerance $A_{22}$. However, it is not very satisfying that amplitudes at resonance peaks of $A_{22}^{*}$ are slightly inconsistent with those of $A_{22}$, for example, the first resonant peak shown in Figure 9. This is because additional damping effects are unavoidably involved when extra mass is installed on the structure and the proposed method only considers removing the mass effects rather than damping effects. Similar observation can also be found in correction of transfer FRF $A_{2-14}^{(2)}$ and point FRF $A_{22}^{(2)}$; see Figures 10 and 11, respectively. It can be seen that the proposed method shows a good effectiveness in resonance frequencies correction. Comparison of frequencies of exact, "measured," and corrected FRFs is given in Table 1. It is shown 


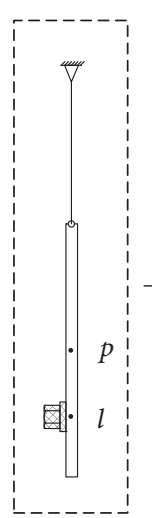

(a1)

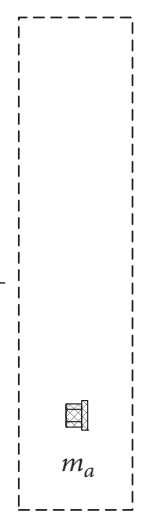

(a2)

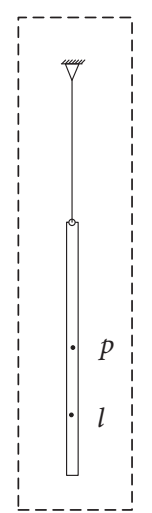

(a3)

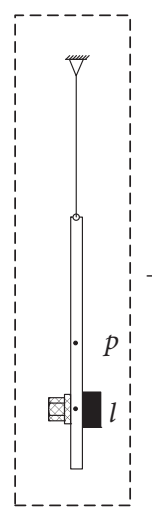

(b1)

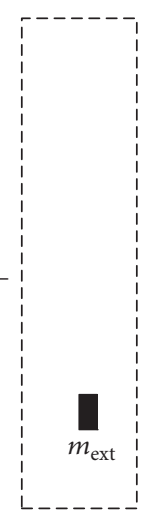

(b2)

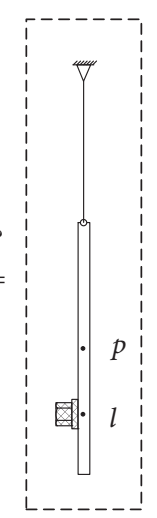

(b3)

(a) Elimination of transducer mass $m_{a}$

(b) Elimination of additional mass $m_{\text {ext }}$

FIGURE 7: Comparative experiments.

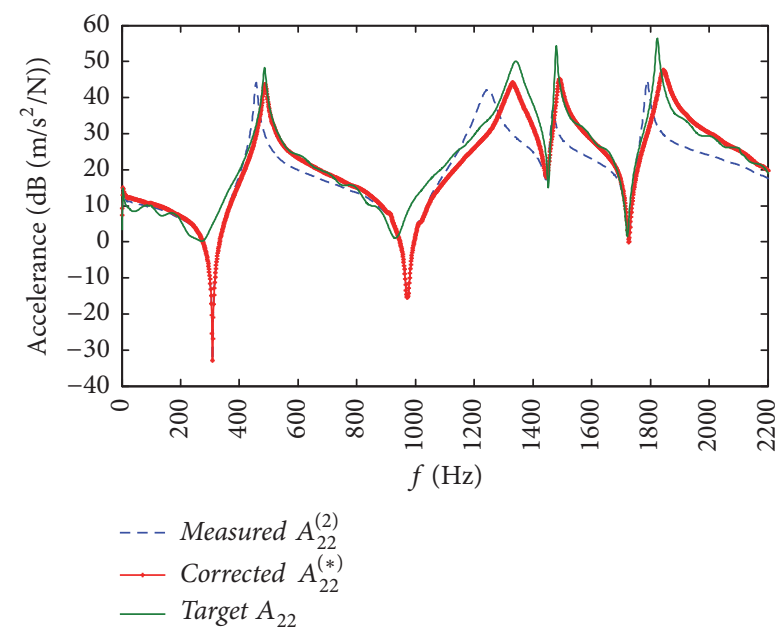

FIGURE 8: Comparison of "measured," corrected, and target FRF $A_{22}$.

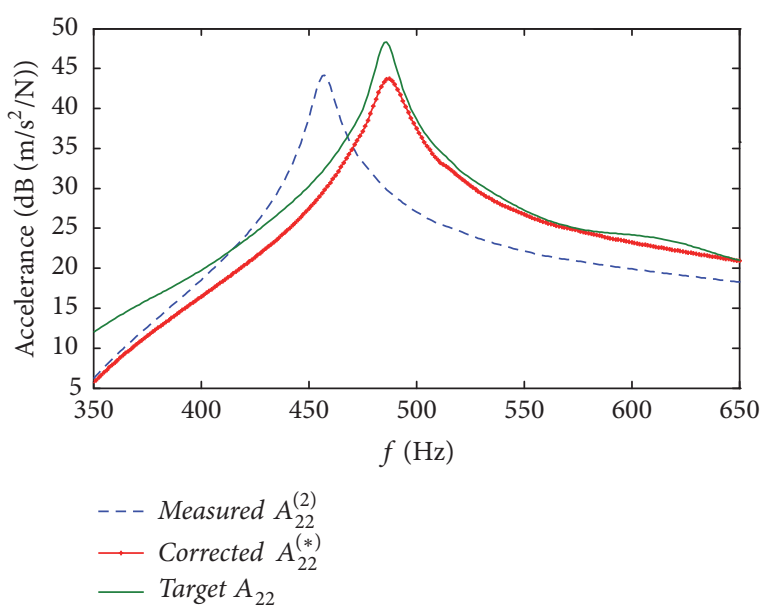

FIgURE 9: Comparison of "measured," corrected, and target FRF $A_{22}$ $(350-650 \mathrm{~Hz})$.

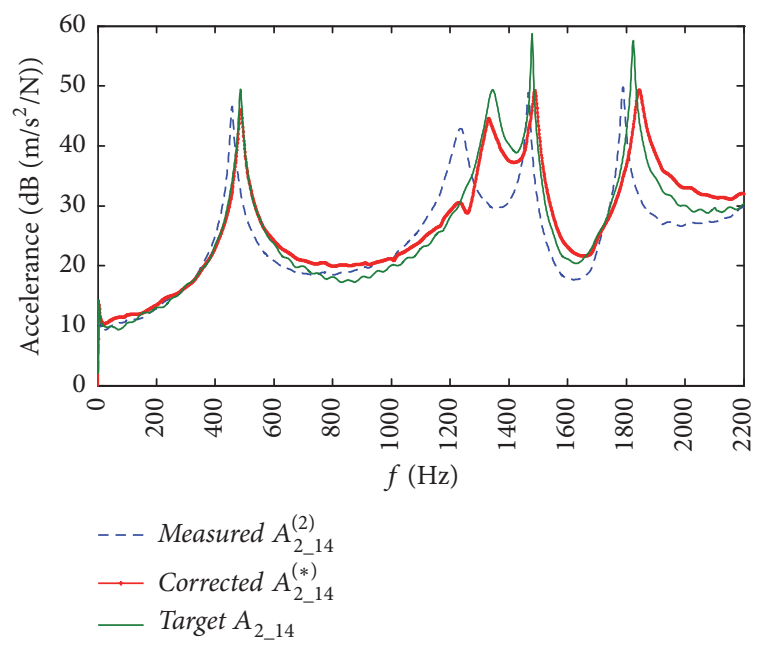

FIGURE 10: Comparison of "measured," corrected, and target FRF $A_{2 \_14}$.

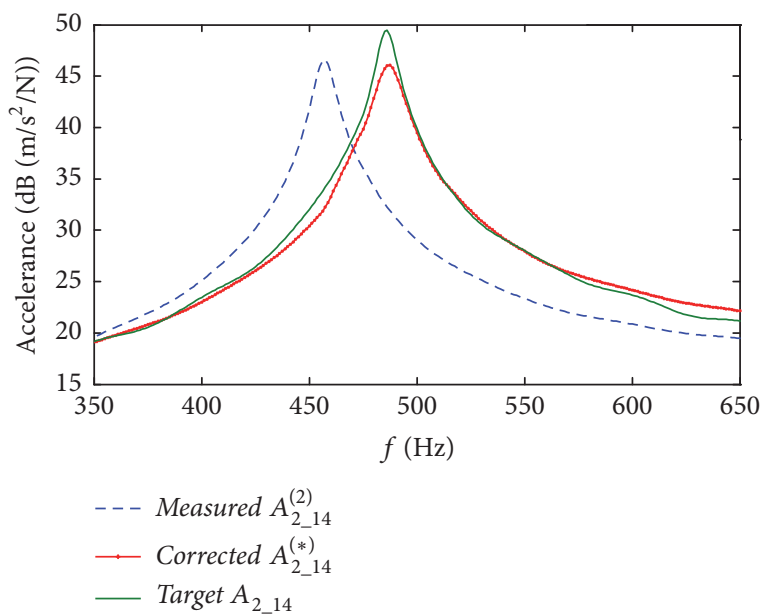

FIGURE 11: Comparison of "measured," corrected, and target FRF $A_{2 \_14}(350-650 \mathrm{~Hz})$. 
TABLE 1: Comparison of frequencies of target, "measured," and corrected FRFs.

\begin{tabular}{|c|c|c|c|c|}
\hline & $\begin{array}{c}1 \mathrm{st} \\
\text { order/Hz }\end{array}$ & $\begin{array}{c}2 \mathrm{nd} \\
\text { order/Hz }\end{array}$ & $\begin{array}{c}\text { 3rd } \\
\text { order/Hz }\end{array}$ & $\begin{array}{c}\text { 4th } \\
\text { order/Hz }\end{array}$ \\
\hline Target & 486.3 & 1341 & 1480 & 1824 \\
\hline Measured & 457.5 & 1246 & 1468 & 1789 \\
\hline Corrected & 487.5 & 1333 & 1490 & 1845 \\
\hline $\begin{array}{l}\text { Errors before } \\
\text { correction }\end{array}$ & $5.9 \%$ & $7.1 \%$ & $0.8 \%$ & $1.9 \%$ \\
\hline $\begin{array}{l}\text { Errors after } \\
\text { correction }\end{array}$ & $0.2 \%$ & $0.6 \%$ & $0.7 \%$ & $1.2 \%$ \\
\hline
\end{tabular}

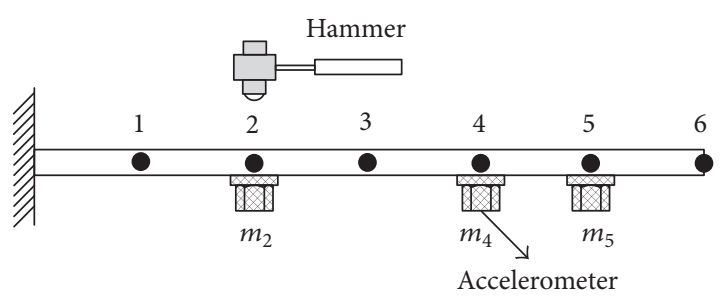

FIGURE 12: Cantilever beam model.

TABLE 2: Parameters of the cantilever beam.

\begin{tabular}{lccccc}
\hline Parameters & $l / \mathrm{m}$ & $b / \mathrm{m}$ & $h / \mathrm{m}$ & $\rho /\left(\mathrm{Kg} \cdot \mathrm{m}^{-3}\right)$ & $E / \mathrm{MPa}$ \\
\hline Values & 1.5 & 0.05 & 0.012 & 7547 & $2.07 \times 10^{5}$ \\
\hline
\end{tabular}

that frequencies errors of 1st and 2nd order are obviously reduced after correction, from $5.9 \%, 7.1 \%$ to $0.2 \%$, and $0.6 \%$, respectively, while frequencies errors of $3 \mathrm{rd}$ and 4 th order are slightly reduced after correction. In particular, for $3 \mathrm{rd}$ order frequency, the error reduction is merely $0.1 \%$ (from $0.8 \%$ to $0.7 \%$ ) after correction. This is due to the fact that the additional mass effects on different orders of modal are not the same. In this test, accelerometer installation position (point 2) coincides with modal node of 3rd order. Then additional mass has little effect on frequency of this order.

\subsection{Numerical Simulation for Multiple Accelerometers Case:} Impact Testing of Cantilever Beam. Simulated hammer impact test of a cantilever beam model is shown in Figure 12 and the physical parameters are given in Table 2. Six points are evenly arranged along the longitudinal direction of the beam. Acceleration sensors with masses of $0.38 \mathrm{Kg}, 0.42 \mathrm{Kg}$, and $0.4 \mathrm{Kg}$ are installed at points 2, 4, and 5, respectively. And the excitation point is chosen to be point 2. Obviously, the measured FRFs $A_{22}^{\prime}, A_{42}^{\prime}$, and $A_{52}^{\prime}$ are affected by the mass effects of accelerometers $\left(m_{2}, m_{4}\right.$, and $\left.m_{5}\right)$ and need to be corrected.

According to (19), additional FRFs $A_{44}^{\prime}, A_{55}^{\prime}$, and $A_{45}^{\prime}$ are required to be "measured" for correction of FRFs $A_{22}^{\prime}, A_{42}^{\prime}$, and $A_{52}^{\prime}$ (note that $A_{45}^{\prime}$ equals $A_{54}^{\prime}$ ), as it can be seen in (20). The "measured" accelerances are numerically generated in this simulation. For comparison purpose, exact FRFs $A_{22}^{*}$,
$A_{42}^{*}$, and $A_{52}^{*}$ corresponding with original structure (without attaching transducers) are also numerical calculated.

$$
\begin{aligned}
& {\left[\begin{array}{l}
A_{22} \\
A_{42} \\
A_{52}
\end{array}\right]} \\
& =\left[\begin{array}{ccc}
1-A_{22}^{\prime} \Delta m_{2} & -A_{24}^{\prime} \Delta m_{4} & -A_{25}^{\prime} \Delta m_{5} \\
-A_{42}^{\prime} \Delta m_{2} & 1-A_{44}^{\prime} \Delta m_{4} & -A_{45}^{\prime} \Delta m_{2} \\
-A_{52}^{\prime} \Delta m_{2} & -A_{54}^{\prime} \Delta m_{4} & 1-A_{55}^{\prime} \Delta m_{5}
\end{array}\right] \\
& \cdot\left[\begin{array}{c}
A_{22}^{\prime} \\
A_{42}^{\prime} \\
A_{52}^{\prime}
\end{array}\right] .
\end{aligned}
$$

The correction results and comparisons are shown in Figures 13, 14, and 15. As shown in these figures, resonance frequencies of the "measured" FRFs are lower than those of exact FRFs due to the mass effects of transducers mass effects. And the magnitudes of resonance frequency reduction of each order are different. It demonstrates that transducers bring different degrees of mass effects on different orders [13-15]. In this study, decreases of low-order resonances frequencies are much smaller than those of high-order resonances frequencies. As a result, transducers mass effects can be ignored if only the first order is concerned. However, elimination of transducers mass effects should be taken into consideration if high-order (e.g., the third order) is of interest. After correction, as expected, corrected FRFs $A_{22}, A_{42}$, and $A_{52}$ are in quite good agreement with exact FRFs $A_{22}^{*}, A_{42}^{*}$, and $A_{52}^{*}$ which demonstrates a good performance of the proposed method. Although the method has been confirmed to be quite promising in a simulated test, some aspects of the method need further consideration before it can readily be used in practical modal test. The measured FRFs in a practical test are inevitably polluted by noise to some extent. Further simulations presented here examine the performance of the method in the case of noisy data. The same system given in Figure 12 is utilized again. The difference is that $A_{22}^{\prime}$, $A_{42}^{\prime}, A_{52}^{\prime}, A_{44}^{\prime}, A_{55}^{\prime}$, and $A_{45}^{\prime}$ are numerically incorporated with commonly used white Gaussian noise (zero mean and variance $\delta_{n}^{2}$ ) [15]. The noise level $\gamma$ is defined as

$$
\gamma^{2}=\frac{\delta_{n}^{2}}{\left|H_{i j}(\omega)\right|_{\text {mean }}^{2}}
$$

where $\left|H_{i j}(\omega)\right|_{\text {mean }}$ is the mean absolute value of $H_{i j}(\omega)$ in the frequency range of interest. The noise levels in present study are set to be $0.5 \%$ and $1 \%$, respectively. Since FRFs are complex values, the random noise should be added to real and imaginary parts of the FRFs, respectively.

Comparisons of measured, exact, and corrected FRFs are shown in Figures 8-13. It can be seen from Figures 16, 17, 19 , and 20 (corrections of $A_{22}$ and $A_{42}$ ) that frequencies bands $(15-25 \mathrm{~Hz}, 45-70 \mathrm{~Hz})$ near second and third resonant peak have been effectively corrected, while others including 


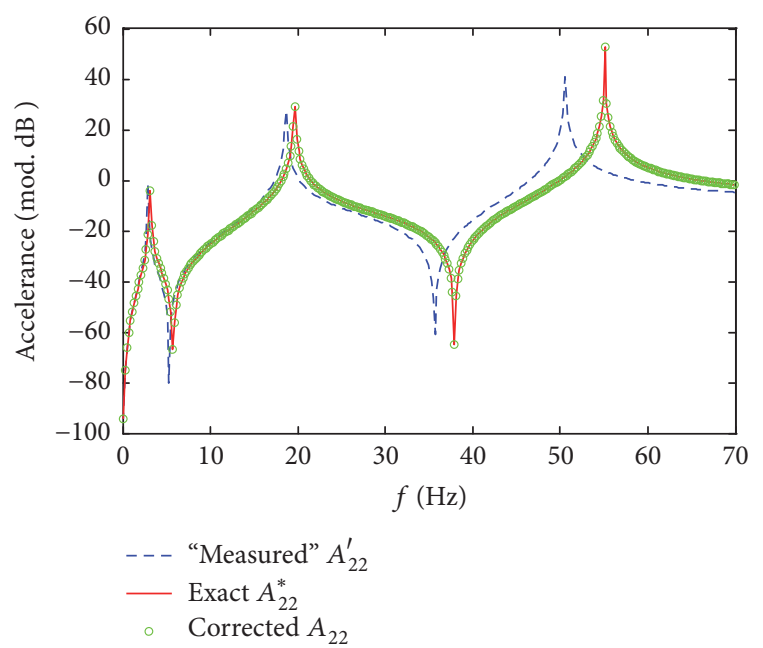

Figure 13: Comparison of "measured," exact, and corrected FRF $A_{22}$.

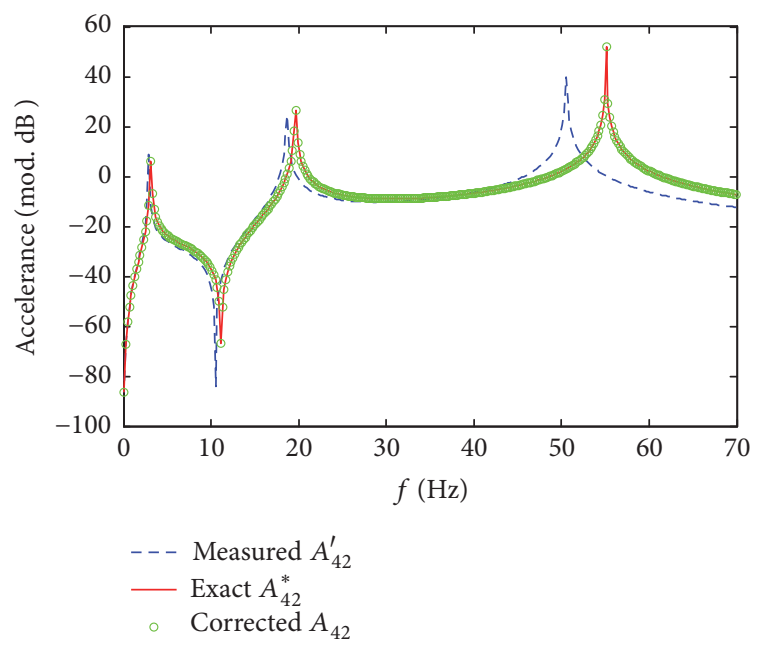

FIGURE 14: Comparison of "measured," exact, and corrected FRF $A_{42}$.

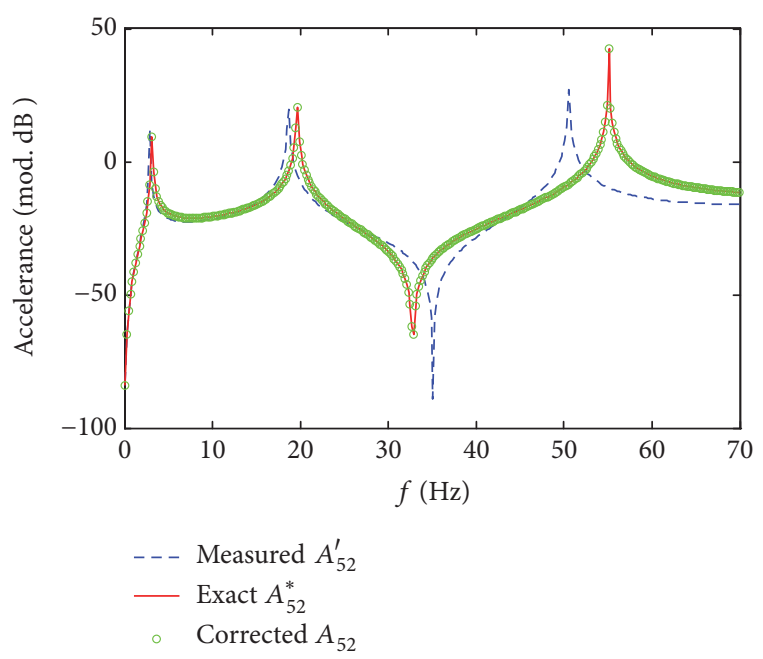

FIGURE 15: Comparison of "measured," exact, and corrected FRF $A_{52}$.

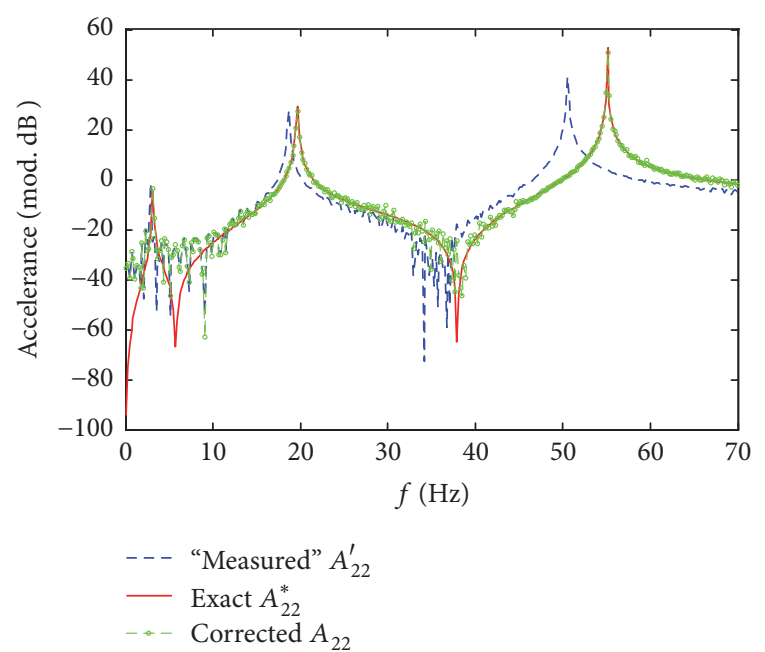

FIGURE 16: Comparison of "measured," exact, and corrected FRF $A_{22}(0.5 \%$ noise $)$.

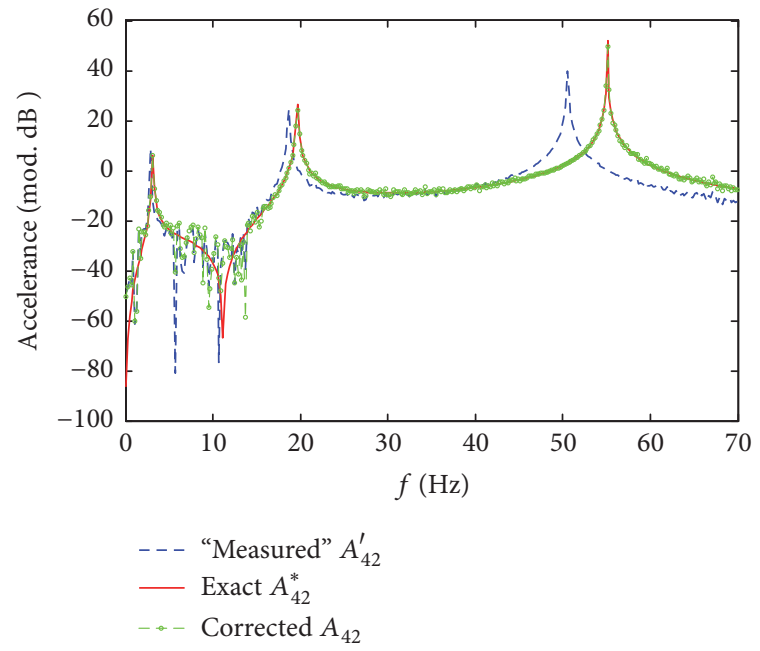

FIGURE 17: Comparison of "measured," exact, and corrected FRF $A_{42}$ (0.5\% noise).

frequencies bands near first resonant peak exhibit poor correction results. In Figures 18 and 21 (correction of $A_{52}$ ), however, the frequencies bands near all the resonant peaks show desirable correction results. This is mainly due to the fact that the frequencies bands of the "measured" FRFs with relatively low amplitudes are greatly polluted by the noise, thus affecting the correction results. Therefore, the proposed correction method may be sensitive to a certain extent to the noise in practical test. In this case, it is suggested that the measured FRFs be preprocessed using the curve-fitting procedure before the proposed correction method is applied. Another possible solution to this problem is to eliminate the noise from the measured FRFs before they are utilized for calculations. 


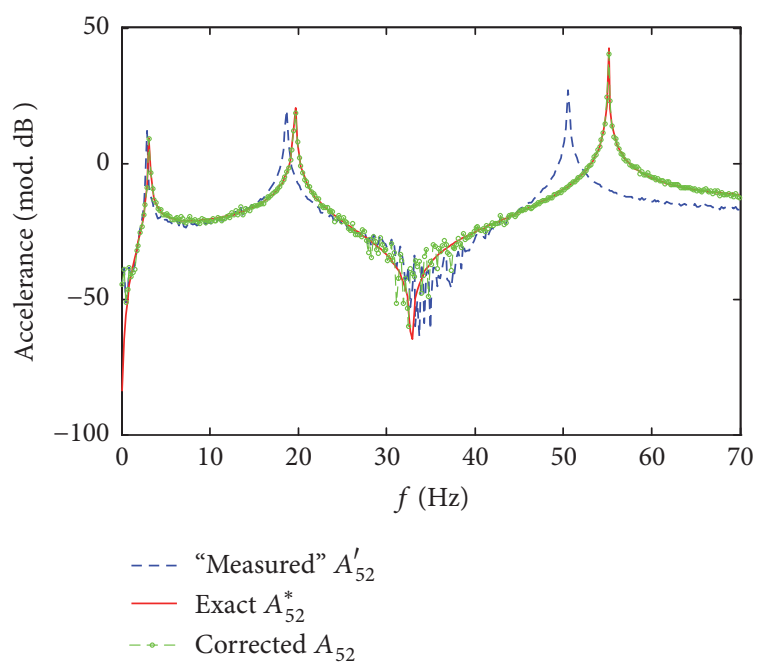

FIGURE 18: Comparison of "measured," exact, and corrected FRF $A_{52}(0.5 \%$ noise $)$.

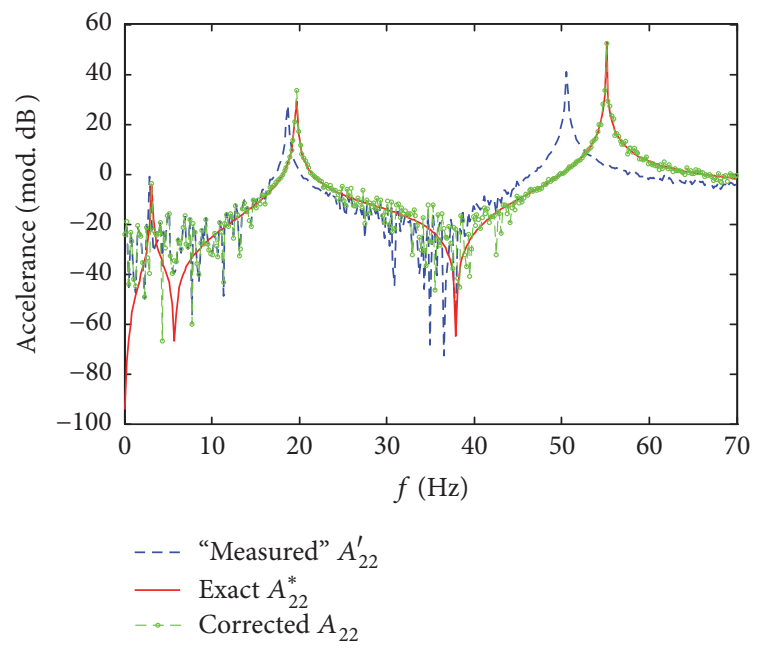

FIGURE 19: Comparison of "measured," exact, and corrected FRF $A_{22}$ (1\% noise).

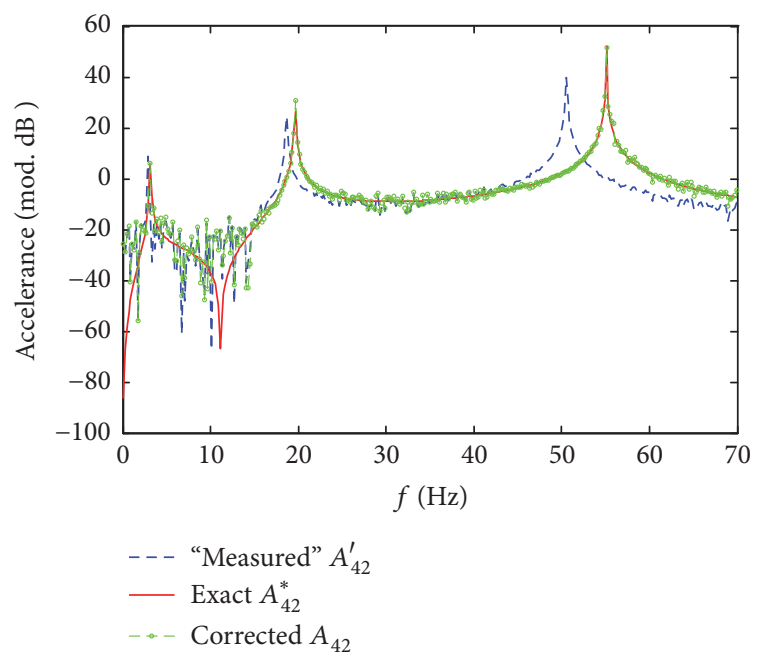

FIgURE 20: Comparison of "measured," exact, and corrected FRF $A_{42}(1 \%$ noise).

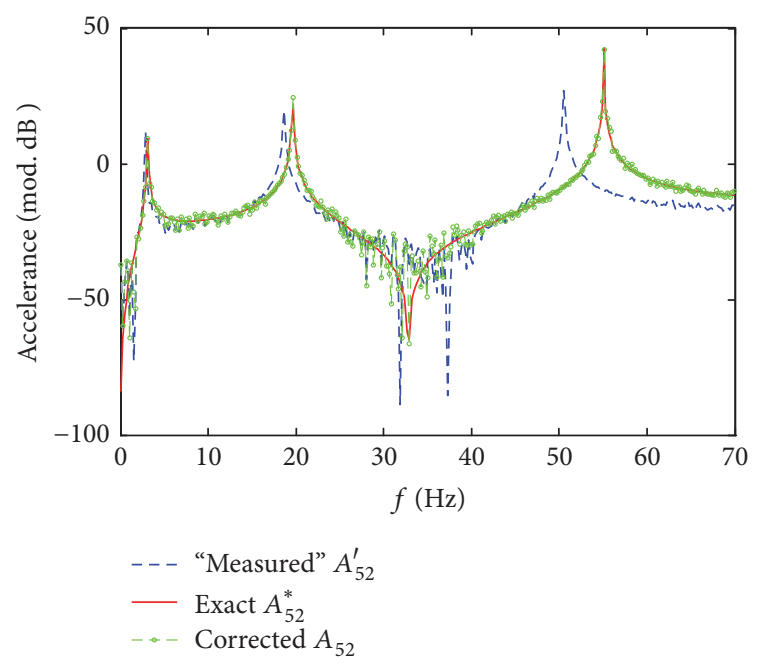

FIGURE 21: Comparison of "measured," exact, and corrected FRF $A_{52}$ ( $1 \%$ noise).

\section{Conclusion}

In modal testing, the measured FRFs are often affected by transducer mass effects. Especially when multiple sensors are employed, transducers mass effects are more significant. This paper deals with removing accelerometers mass effects from the measured FRFs in hammer impacting testing. The research indicates the following:

(1) When $N$ accelerometers are attached at points $1,2, \ldots, N$, respectively, at least $N(N+1) / 2$ FRFs related to all the response points are required to measure for eliminating accelerometers mass effects from the measured FRFs $A_{1 p}^{\prime}, A_{2 p}^{\prime}, \ldots, A_{N p}^{\prime}$ (subscript $p$ denotes excitation point).

(2) Advantage of this method is that additional configurations of the structure for FRFs measurements (used for correction) are not required, such as moving the sensor to different response locations, adding extra sensors or dummy masses on the structure.

The proposed method shows good effectiveness in experimental validation for single accelerometer case. A simple numerical example presented also demonstrates a good performance in the numerical simulation for multiple accelerometers case. However, further simulations with noisy-polluted data show that the accuracy of correction results will be affected to some extent by the noise. It is suggested that the measured FRFs be preprocessed using the curve-fitting procedure or noise reduction processing before applying this method. Since the FRFs measurements required for corrections refer to different excitation points, moving the exciter to various locations is required. This is usually easy to achieve in hammer impact testing. However, moving the exciter is usually inconvenient (if not impossible) in shaker modal testing because the excitation point is usually fixed in such case. Therefore, the proposed method is more applicable for hammer impact test. Further work is necessary to extend 
this proposed method so as to make it also applicable for shaker modal test case. This does not mean "to make the shaker to be movable" but rather attempts to obtain the FRFs with different excitation points by employing the FRFs with same excitation points. Thus, all the FRFs required for correction can then be obtained by using the FRFs with same excitation points which avoids troublesome movements of the shaker. However, it will probably involve additional FRFs measurements with extra dummy masses or accelerometers attached.

\section{Conflicts of Interest}

The authors declare that they have no conflicts of interest.

\section{Acknowledgments}

This work was supported by Hubei Provincial Natural Science Foundation of China (2017CFB128) and Hubei Provincial Department of Education Scientific Research Project (Q20171405).

\section{References}

[1] D. J. Ewins, Modal Testing: Theory, Practice and Applications, Research Studies Press, England, UK, 2nd edition, 2000.

[2] D. J. Ewins, Modal Testing: Theory and Practice, Research Studies Press, England, UK, 1984.

[3] H. Koruk, "Quantification and minimization of sensor effects on modal parameters of lightweight structures," Journal of Vibroengineering, vol. 16, no. 4, pp. 1952-1963, 2014.

[4] Z. Zhang, P. Zhu, and X. Bao, “The mass loading effect on lightweight cantilever mode frequency measurement by optical fiber sensor," in Proceedings of the SPIE - The International Society for Optical Engineering, Montreal, Canada, June 2008.

[5] J. Decker and H. Witfeid, "Correction of transducer-loading effects in experimental modal analysis," in Proceedings of the 13th International Modal Analysis Conference, 1995.

[6] M. R. Ashory, "Correction of mass-loading effects of transducers and suspension effects in modal testing," in Proceedings of the International Modal Analysis Conference, pp. 815-828, Orlando, FL, USA, February 1998.

[7] O. Cakar and K. Y. Sanliturk, "Elimination of transducer mass loading effects from frequency response functions," Mechanical Systems and Signal Processing, vol. 19, no. 1, pp. 87-104, 2005.

[8] J. Ren and S. Bi, "Identification and correction of force transducer mass effects in modal testing," Journal of Vibration and Shock, vol. 33, no. 14, pp. 112-117, 2014.

[9] S. Bi, J. Ren, W. Wang, and G. Zong, "Elimination of transducer mass loading effects in shaker modal testing," Mechanical Systems and Signal Processing, vol. 38, no. 2, pp. 265-275, 2013.

[10] P. Zamani, A. Taleshi Anbouhi, M. Ashory, M. Khatibi, and R. Masoudi Nejad, "Cancelation of transducer effects from frequency response functions: experimental case study on the steel plate," Advances in Mechanical Engineering, vol. 8, no. 4, pp. 1-12, 2016.

[11] T. Yin, "Elimination of transducer additional mass effects in time-domain responses," Journal of Vibration and Shock, vol. 35, no. 4, pp. $28-34,2016$.
[12] J. Ren, J. Wang, and X. Zhou, "Correction of multiple transducers masses effects from the measured FRFs," in Proceedings of the ASME 2017 International Design Engineering Technical Conferences and Computers and Information in Engineering Conference, IDETC/CIE 2017, Cleveland, OH, USA, 2017.

[13] J. Ren, J. Wang, X. Zhou, J. Sun, Q. Wang, and K. Ting, "A Quick Method for Assessing Transducer Mass Effects on the Measured FRFs," in Proceedings of theASME 2016 International Design Engineering Technical Conferences and Computers and Information in Engineering Conference, IDETC/CIE 2016, pp. V8T-V10T, Charlotte, NC, USA, 2016.

[14] N. H. Baharin and R. Abdul Rahman, "Effect of accelerometer mass on thin plate vibration," Jurnal Mekanikal, vol. 29, pp. 100111, 2009.

[15] J. Ren and J. Wang, "Assessment of transducer mass effects on measured FRFs in shaker modal testing," Journal of Vibroengineering, vol. 19, no. 5, pp. 3472-3487, 2017. 


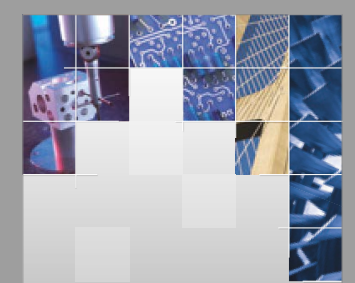

\section{Enfincering}
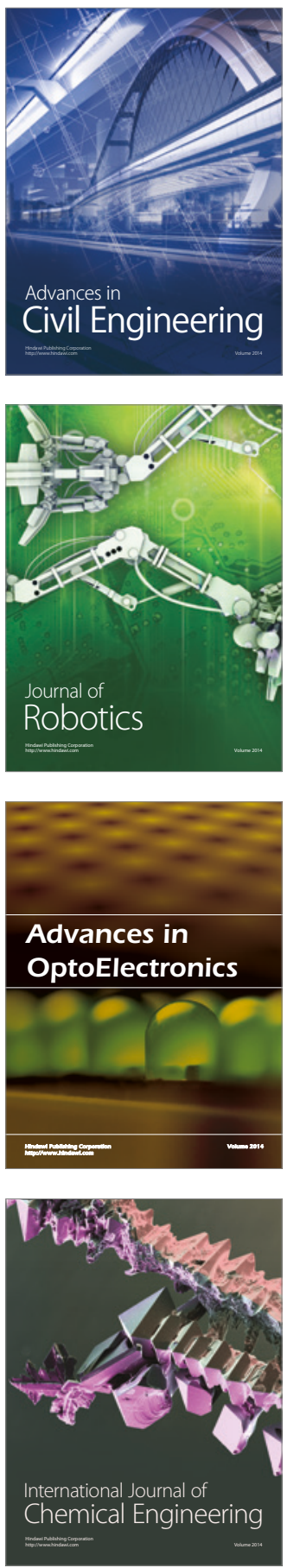

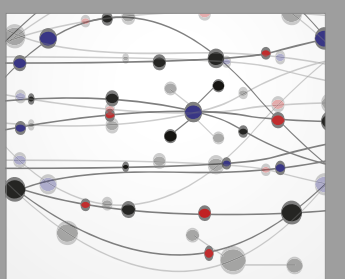

The Scientific World Journal

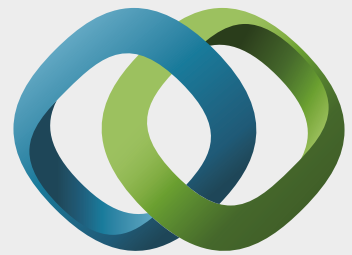

\section{Hindawi}

Submit your manuscripts at

https://www.hindawi.com
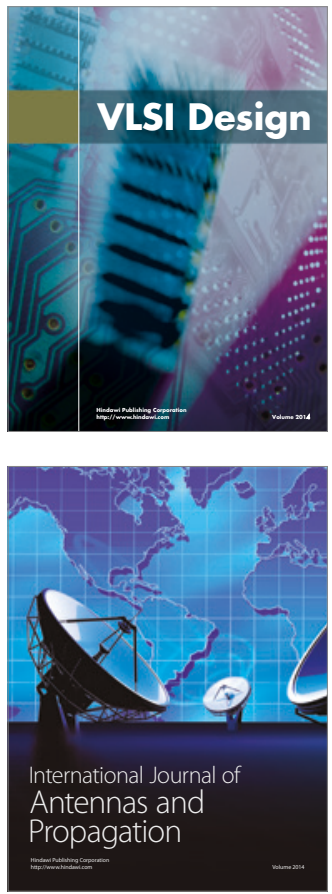

\section{Rotating}

Machinery
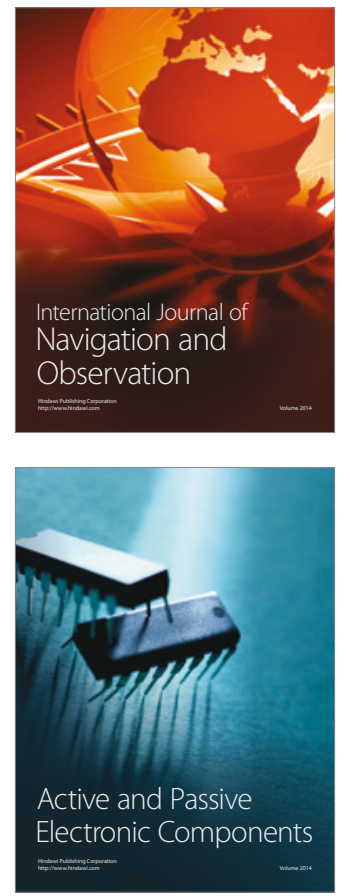
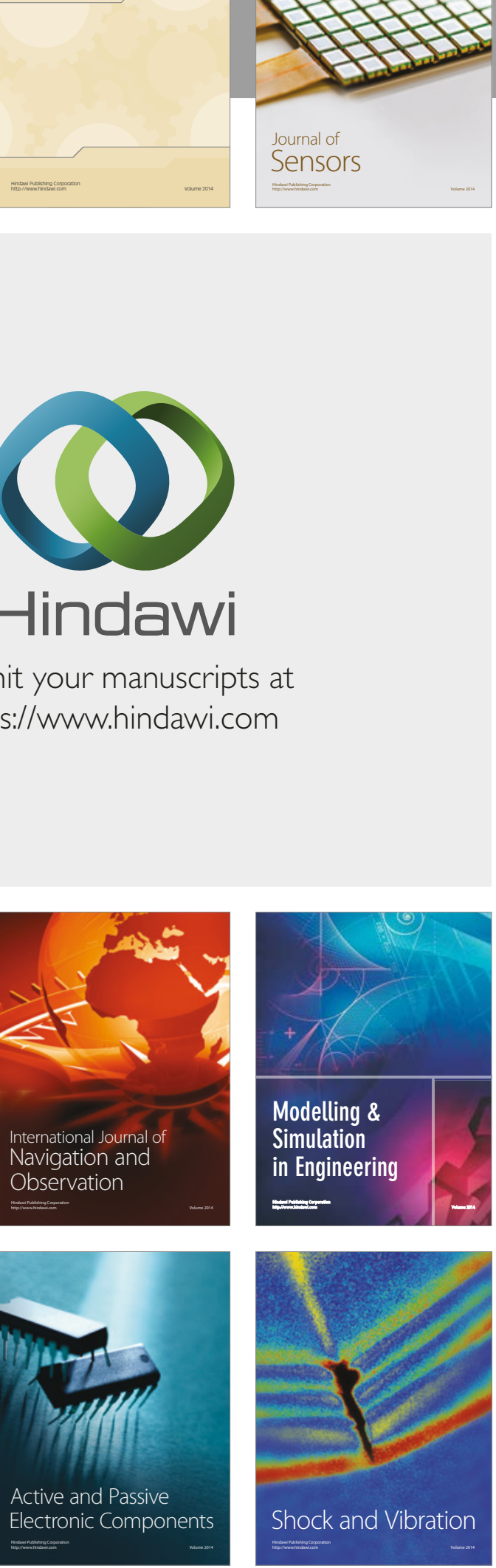
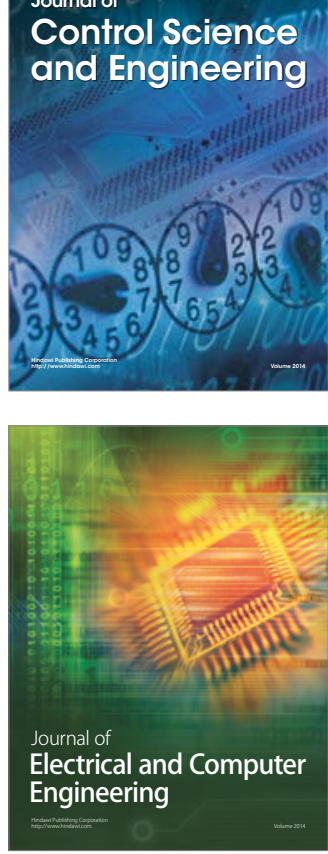

Distributed

Journal of

Control Science

and Engineering
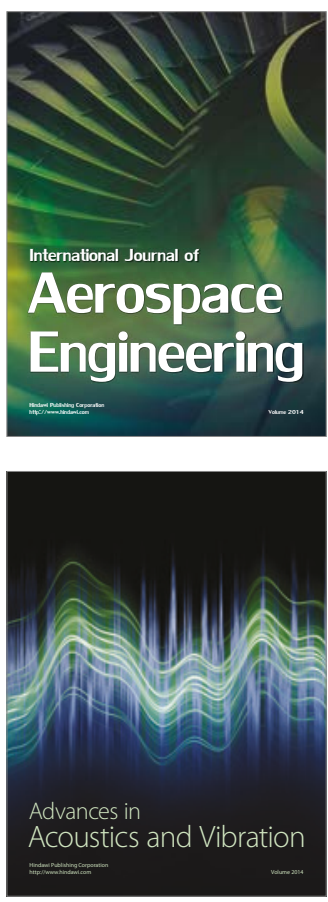

Sensor Networks 